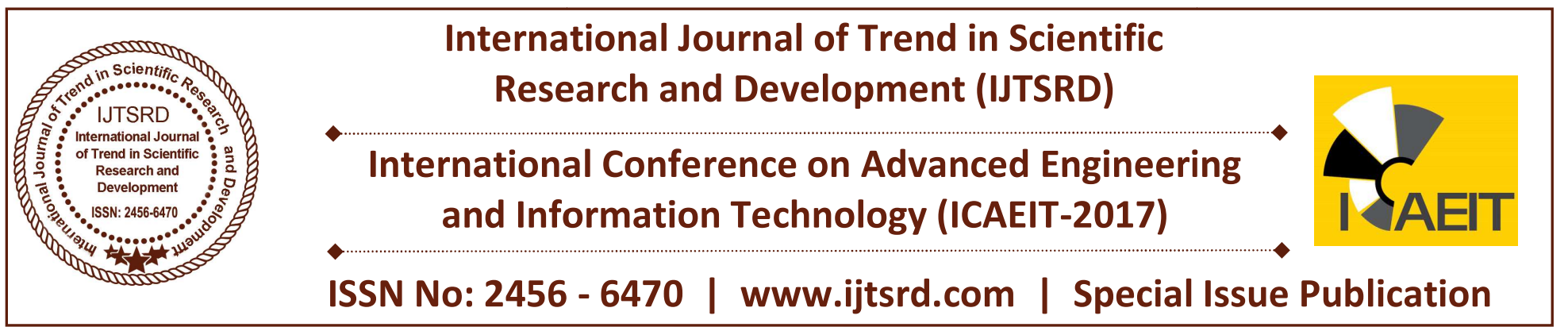

\title{
Wideband Width Impedance Matching Topologies for PA Applications
}

\author{
Vinukumar Luckose ${ }^{1}$, Veeraiyah Thangasamy ${ }^{1}$, Vinesh Thiruchelvam ${ }^{1}$, \\ Sajitha Smiley². Shaina Suresh ${ }^{2}$, \\ ${ }^{1}$ Asia Pacific University of Technology and Innovation, 57000, Kuala Lumpur, Malaysia \\ ${ }^{2}$ Linton University College, Mantin, Negeri Sembilan, Malaysia
}

\section{ABSTRACT}

In this paper, the design of a wideband width impedance matching topology for Power Amplifier (PA) applications has introduced. The design topology has implemented with single, double and triple stages to demonstrate the wideband width frequency range for input and output matching network section of the PA amplifier. Each stages of the proposed matching circuit has LC filter with high and low pass design configuration. The single stage configuration provides the wide band frequency of $1.07 \mathrm{GHz}$ to $2.094 \mathrm{GHz}$ at $-74 \mathrm{~dB}$ gain. The frequency range of $-0.75 \mathrm{GHz}$ to $2.95 \mathrm{GHz}$ has obtained in double stage configuration with $-28 \mathrm{~dB},-92 \mathrm{~dB}$ and $40 \mathrm{~dB}$ gain. The triple stage offers a wide frequency range of $0.59 \mathrm{GHz}$ to $3.7 \mathrm{GHz}$ with $-17 \mathrm{~dB},-33 \mathrm{~dB}$, $96 \mathrm{~dB},-55 \mathrm{~dB}$ and $-30 \mathrm{~dB}$ gain at the cut off frequency of $1.5 \mathrm{GHz}$ which is suitable for $\mathrm{PA}$ applications.

Keywords: Wideband, Power Amplifier, Impedance Matching Circuit, Low Pass filter, high Pass Filter.

\section{INTRODUCTION}

$\mathrm{RF}$ and microwave power amplifiers are widely used in all radio communications such as; radar, guidance, mobile and electronic compact systems as an essential part of the transmitter and receiver chain for providing the required frequency signal [1]. The wireless communication system has different communication identification of standards for various applications, such as worldwide interoperability for microwave access (WiMAX), HSPA, HSPA+, 3G WCDMA and 4G long term evolution (LTE) [2-3].
The modern high-data-rate communication systems required wide band frequency range for the PA to support different wireless standards [4]. However, the conventional narrow-band LNAs has poor performance to meet the latest wireless standards [5]. The narrow- band multimode transceivers are frequently implemented with some narrow band PAs for each frequency band, which increases the chip area and the cost [6]. Wideband width frequency signal has received much attention in the recent wireless communication system due to its good signal quality to satisfy the different standards at the same time [5]. The new generation wireless communication system deployed $800 \mathrm{MHz}$ to $3 \mathrm{GHz}$ range of frequency for various applications [7]. Few methods are implemented to provide wide band frequency for communication signals by other researchers. The twostage broad band power amplifier [6] provides 0.7 to $1.5 \mathrm{GHz}$ wide band frequency with $16.3 \mathrm{~dB} 18 \mathrm{~dB}$ power gain. The Doherty power amplifier design offers 1.8 to $3.6 \mathrm{GHz}$ wideband frequency with $6 \mathrm{~dB}$ power gain [7]. The broad band amplifier using low pass bias network [8] gives 1.8 to $2.7 \mathrm{GHz}$ with 43.5 $\mathrm{dB}-45 \mathrm{~dB}$ power gain. The improved Doherty amplifier with minimum phase delay in output matching network [9] has designed at 1.6 to $2.2 \mathrm{GHz}$ with $36 \mathrm{~dB}$ power gain. The wideband Doherty amplifier using peaking output matching network [10] provides 2.3 to $2.8 \mathrm{GHz}$ wide band frequency at -49 $\mathrm{dBc}$ linearity gain. In the literature various method have been proposed to improve band width. But the range of frequency is within certain level of frequency. 
In this paper input and output impedance matching network topologies has designed for power amplifier application to obtain wide band frequency. The proposed work is designed with single, double and triple stage impedance matching circuit with HighLow and Low- High cascaded configuration. Each stages are embedded with high pass and low pass filter network. The triple stage impedance matching topology achieved with 0.6 to $3.75 \mathrm{GHz}$ wide band frequency.

\section{Design Methodology:}

Figure 1 shows the block diagram of basic impedance matching network configuration with power amplifier stage. In the high-low input-matching network, the transformation ratio has $50 \Omega$ to $1.85 \Omega$ while the output-matching network low-high used with $1.85 \Omega$ to $50 \Omega$. The input and output matching network has designed with the combination of High and Low pass cascaded configuration.

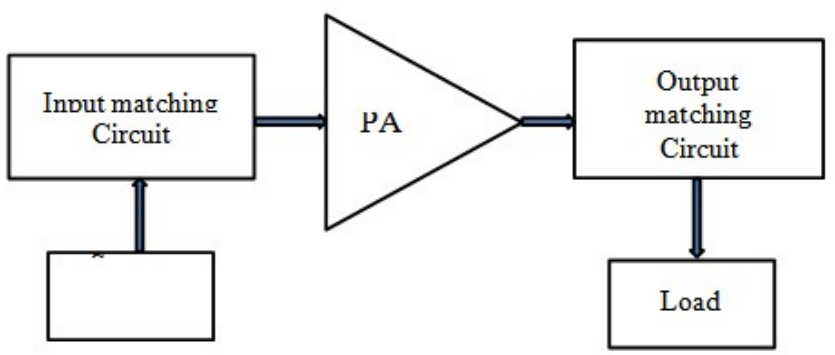

Figure1. Basic Impedance Matching Block Diagram

\subsection{High-Low cascaded network design for Input matching circuit:}

The single stage high-low impedance matching network is shown in Figure 2. The components L1 and $\mathrm{C} 1$ act as high pass filter and L2, C2 act as Low pass filter. The source $\left(R_{\mathrm{s}}\right)$ has the impedance of $50 \Omega$ and the load resistance is $1.85 \Omega$. In the single stage, the matching levels are thus $50 \Omega, 9.62 \Omega$ and $1.85 \Omega$. The impedance transformation ratio is 27 to one and this is achieved by cascading the high and low pass impedance transformation to one. The value of inductor (L) and capacitor (C) has designed using the matching levels of the circuit. The impedance matching calculations has done at a center of frequency of $1.5 \mathrm{GHz}$. Figure 3 shows the double stage impedance matching network. The L1, L2 and C1, C2 act as high pass filter; L3, L4 and C3, C4 act as low pass filter. The impedance matching levels are thus $50 \Omega, 21.92 \Omega, 9.61 \Omega, 4.21 \Omega$ and $1.85 \Omega$ used in double stage matching network. Triple stage highlow impedance matching network is shown in Figure 4. The circuit has designed with three high pass filter cascaded with another three-stage low pass filter. The impedance matching levels has designed with the impedance transformation from $50 \Omega$ to $1.85 \Omega$. In three stage cascaded network the matching levels are thus $50 \Omega, 28.82 \Omega, 16.66 \Omega, 9.62 \Omega, 5.55 \Omega 3.207 \Omega$ and $1.85 \Omega$.

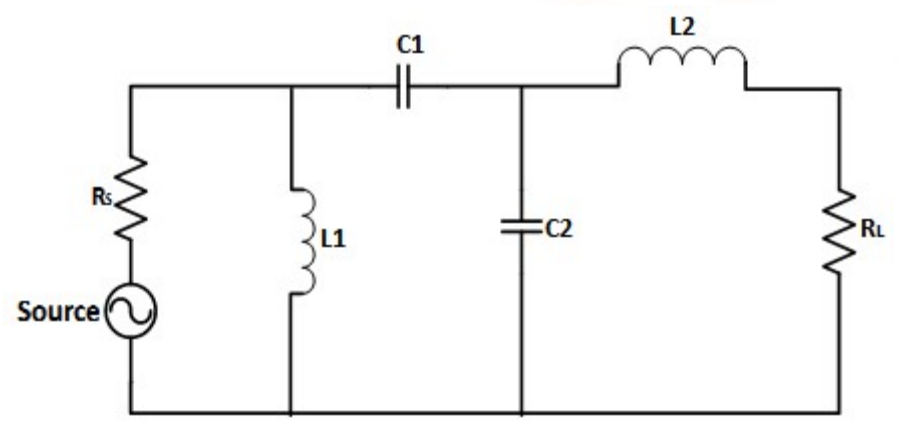

Figure2. Single stage High - Low Matching Circuit

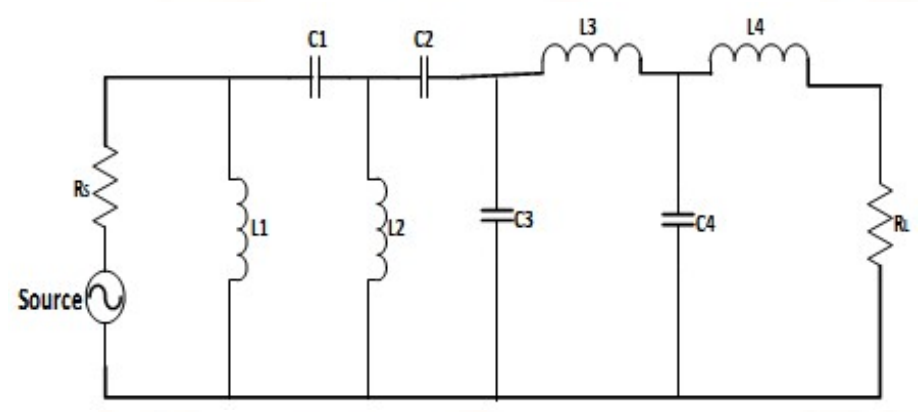

Figure3. Double stage High - low matching circuit

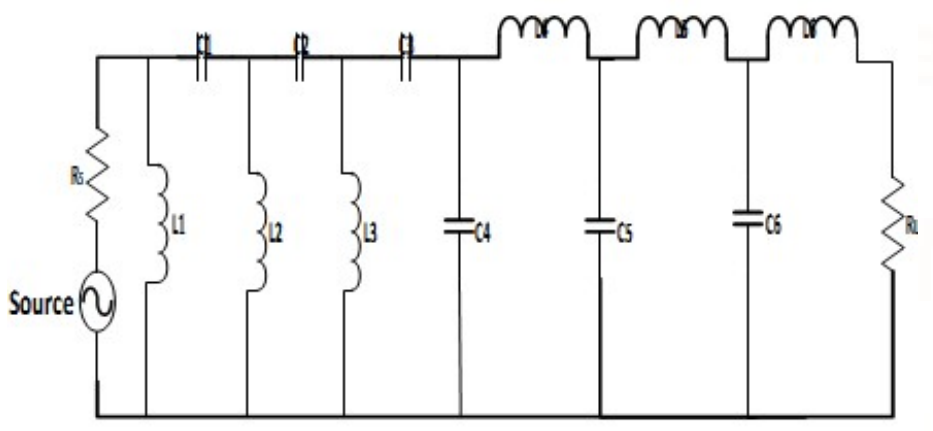

Figure4. Triple stage High-Low matching Circuit

\subsection{Low-High cascaded network design for output matching circuit:}

The design steps for Low-High impedance matching networks has discussed below. The impedance matching network shown in Figure 5 is the single stage network with the components $\mathrm{L} 1$ and $\mathrm{C} 1$ act as low pass filter and L2, C2 act as high pass filter. The transformation ratio has $50 \Omega$ to $1.85 \Omega$. The matching network has designed with the same value of $\mathrm{Q}$ as used in High-low matching network. The matching levels are thus $1.85 \Omega, 9.62 \Omega$ and $50 \Omega$. The impedance matching calculations has done at a 
center of frequency of $1.5 \mathrm{GHz}$. The double stage low- high impedance matching network has shown in Figure6. The L1, L2 and C1, C2 act as low pass filter; L3, L4 and C3, C4 act as high pass filter. The impedance matching levels are thus $1.85 \Omega, 4.21 \Omega$, $9.61 \Omega, 21.92 \Omega$ and $85 \Omega$ used in double stage matching network. Figure 7 shows the Triple stage low-high impedance matching network. Three low pass filter cascaded with another three-stage high pass filter for obtaining the wideband frequency. The matching levels are thus $1.85 \Omega, 3.20 \Omega, 5.55 \Omega, 9.62$ $\Omega, 16.6 \Omega, 28.82 \Omega$ and $50 \Omega$ used in triple stage matching network.

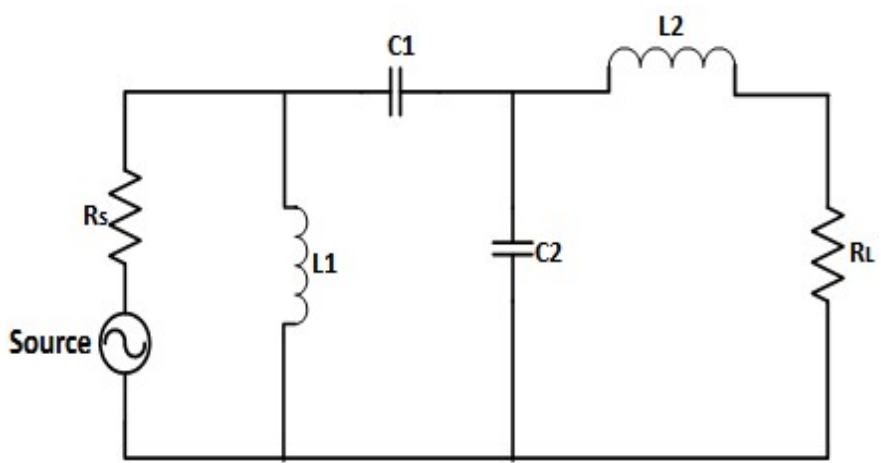

Figure5. Single stage Low - High Matching Circuit

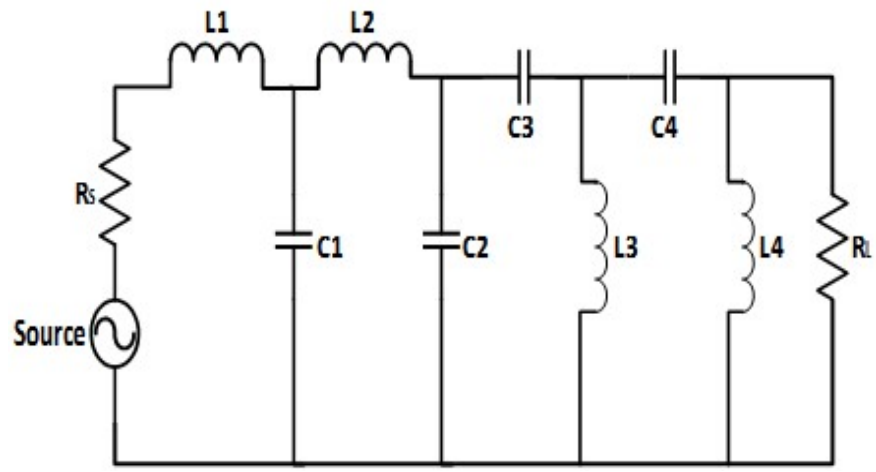

Figure6. Double stage Low - High Matching Circuit

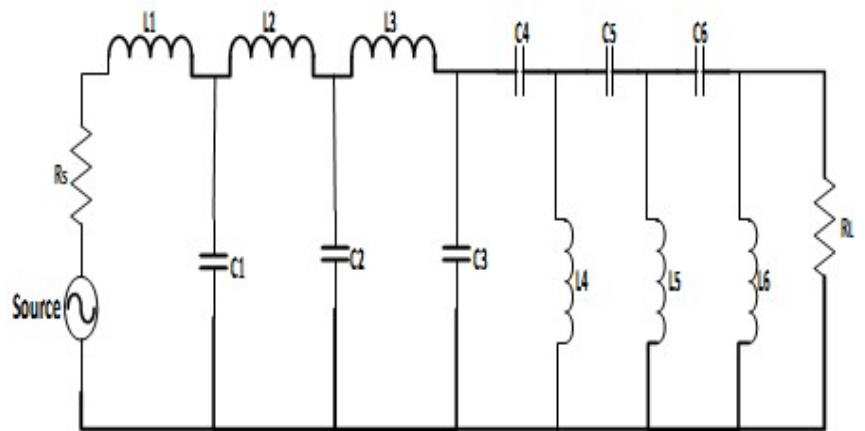

Figure7. Triple stage Low - high Matching Circuit

\section{Results and Discussion}

The performance of the various wide bandwidthmatching topologies has evaluated and the simulated results using Cadence Software has discussed below.

\subsection{Simulation Results for High-Low Cascaded Network for input matching circuit:}

In this section the High-Low impedance matching network for single, double and triple stage has discussed. The single stage High-Low matching circuit is shown in Figure 8. The matching circuit has achieved a widebandwith frequency range of $1.2 \mathrm{GHz}$ to $2.2 \mathrm{GHz}$ with the cut of frequency of $1.5 \mathrm{GHz}$. The gain of the single stage matching circuit has $-73 \mathrm{~dB}$. The $\mathrm{Q}$ factor value for the single stage has 2.048. The Double stage High-Low matching circuit as shown in Figure 9 has obtained the wide band frequency band of $0.7 \mathrm{GHz}$ to $2.9 \mathrm{GHz}$ with the same cut off frequency. The gain values are obtained at $-29 \mathrm{~dB},-93$ $\mathrm{dB}$ and $-47 \mathrm{~dB}$ with the cut off frequency of $1.5 \mathrm{GHz}$. The larger wide band frequency has obtained in the triple stage impedance matching circuit as shown in Figure 10. The range of frequency is from $0.6 \mathrm{GHz}$ to $3.75 \mathrm{GHz}$ with the gain values of $-18 \mathrm{~dB},-33 \mathrm{~dB},-98$ $\mathrm{dB},-57 \mathrm{~dB}$ and $-31 \mathrm{~dB}$ respectively. In triple stage high-low matching circuit the frequency band is wider and the gain value is higher. The value of $Q$ factor is found as 0.855 which lower than the single and double stage matching circuit.

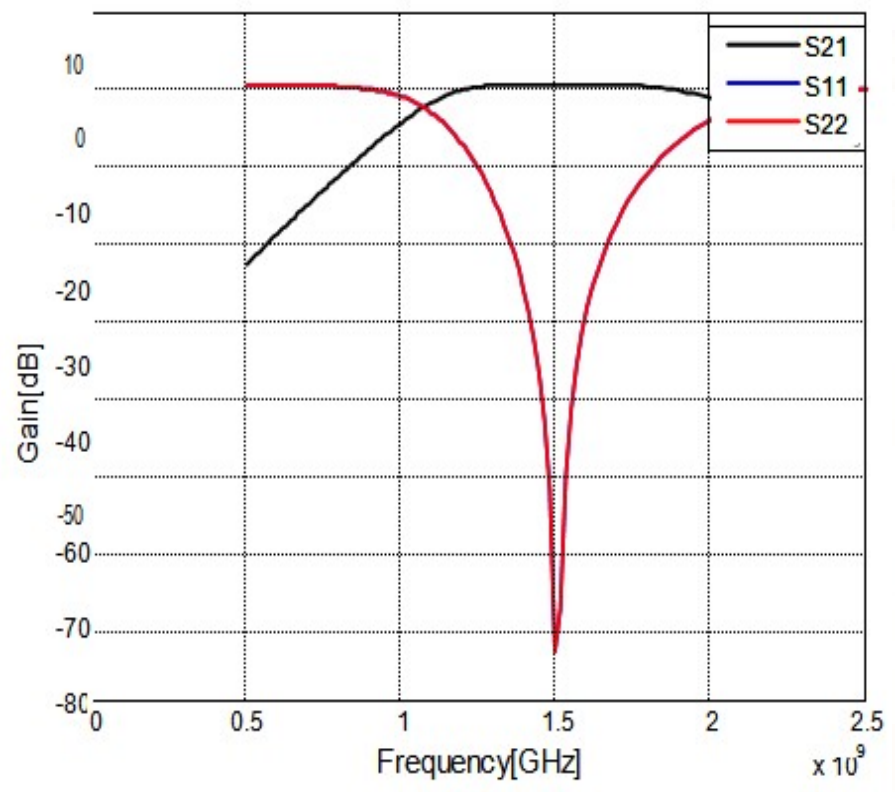

Figure8. Frequency and gain for sinlge stage HighLow matching circuit 
International Journal of Trend in Scientific Research and Development (IJTSRD) | ISSN: 2456-647

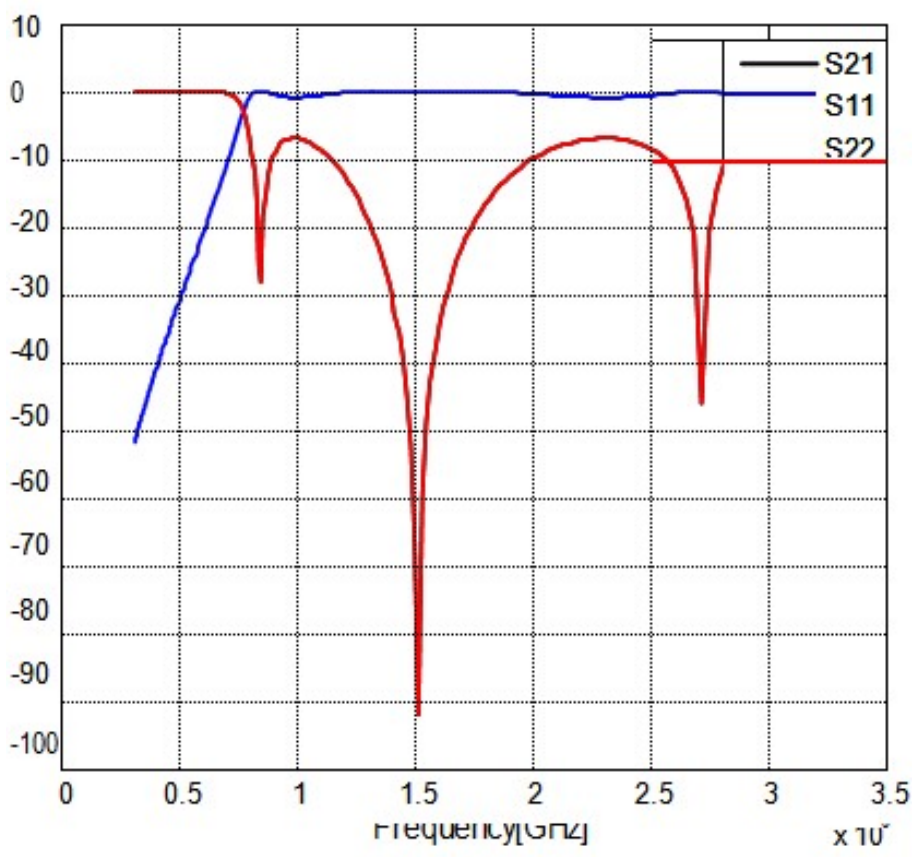

Figure9. Frequency and gain for double stage HighLow Matching Circuit

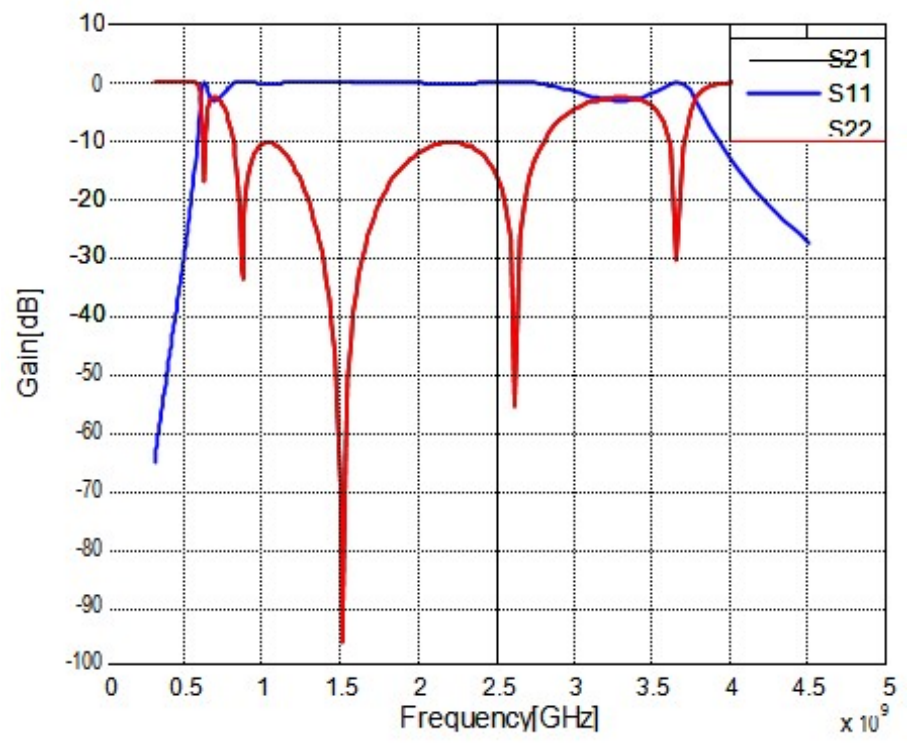

Figure10. Frequency and gain for Trple stage HighLow Matching Circuit

\subsection{Simulation Results for Low-High Cascaded}

Network for output matching circuit:

In this section the Low-High impedance matching network for single, double and triple stage has discussed. Figure 11 shows the frequency and gain for the single stage Low-High matching circuit. The designed matching circuit has achieved a wideband with frequency range of $1.2 \mathrm{GHz}$ to $2.2 \mathrm{GHz}$ with the cut of frequency of $1.5 \mathrm{GHz}$. The gain of the single stage matching circuit has $-73 \mathrm{~dB}$. The Low-High single, double and triple stage matching circuit has a similar results with the High-Low matching circuits.

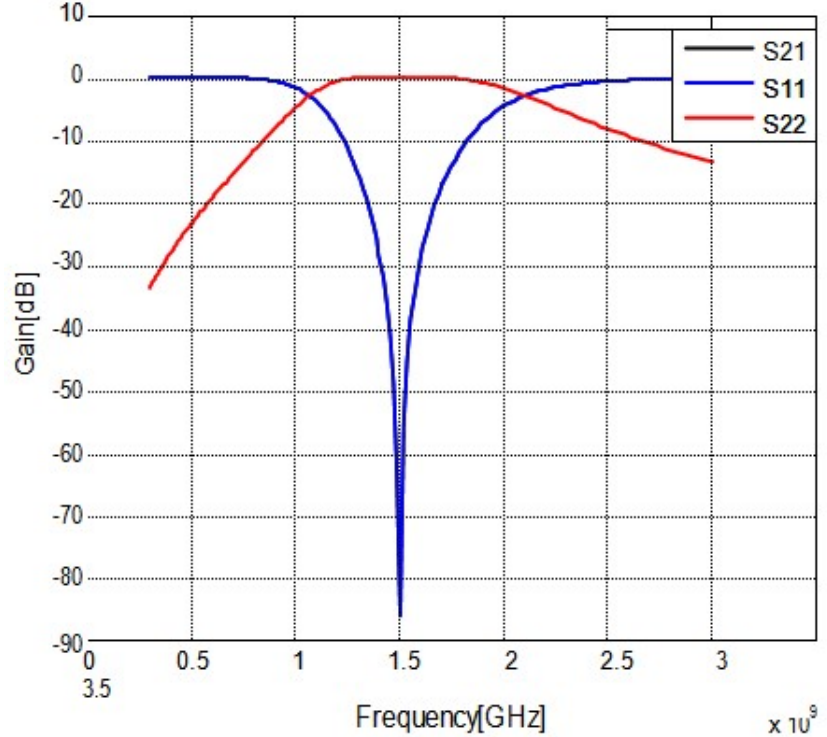

Figure11. Frequency and gain for sinlge stage LowHigh matching circuit

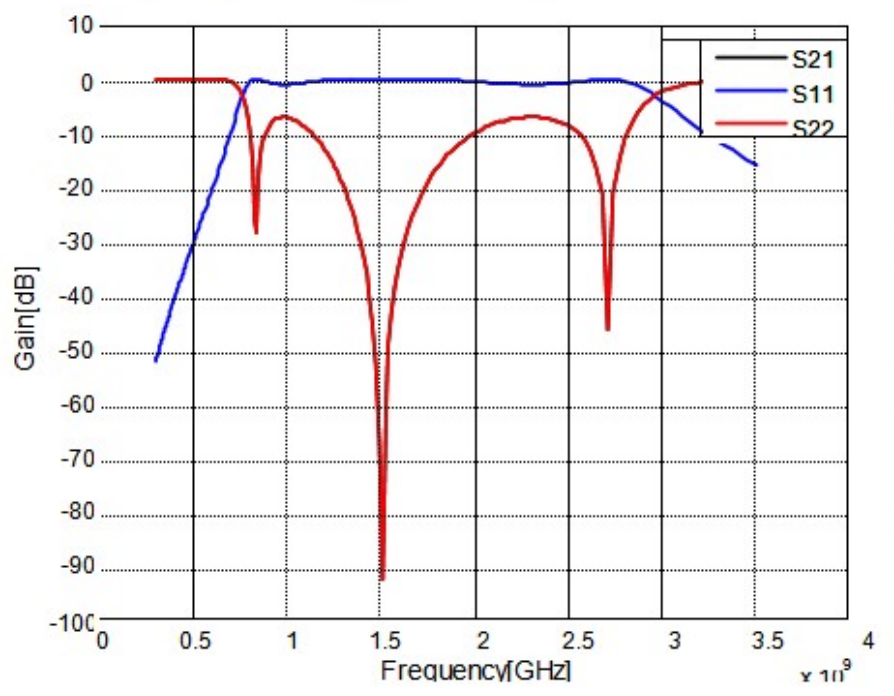

Figure12. Frequency and gain for double stage LowHigh matching circuit

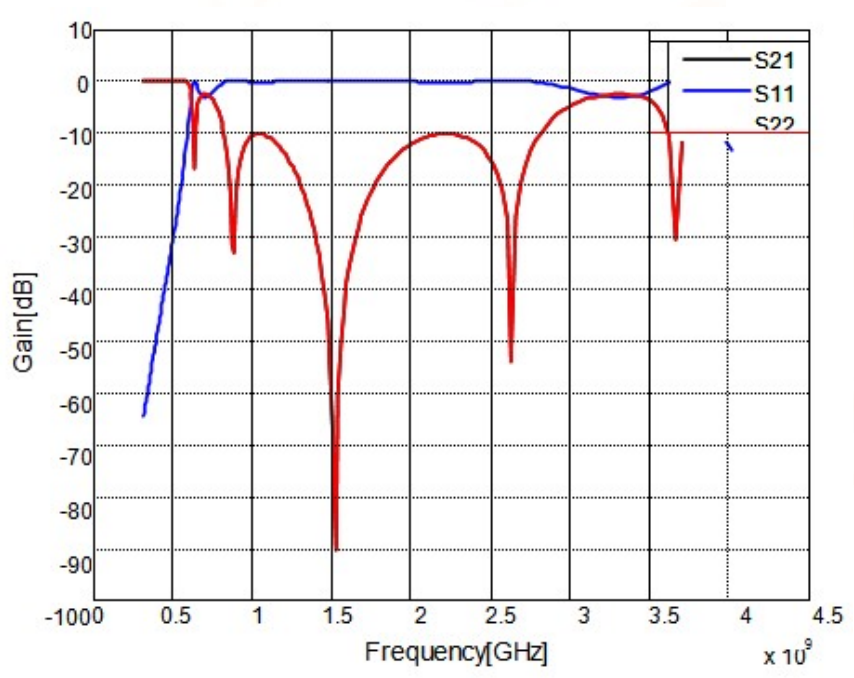

Figure13. Frequency and gain for Triple stage Low-High matching circuit 


\section{Conclusion:}

This paper present a wideband width impedance matching networks for PA applications. The proposed topologies has designed for input matching and output matching circuit for Power amplifier. Three various stages; single, double and triple stage has implemented in each section in order to differentiate the wide band width frequency. The single stage wide band width impedance circuit has achieved with 1.2 $\mathrm{GHz}$ to $2.2 \mathrm{GHz}$ frequency range with gain of $-73 \mathrm{~dB}$. The double stage impedance matching circuit provide the frequency range of $0.7 \mathrm{GHz}$ to $2.9 \mathrm{GHz}$. The gain of $-29 \mathrm{~dB},-93 \mathrm{~dB}$ and $-47 \mathrm{~dB}$ has achieved in the double stage topology. The wider frequency of 0.6 $\mathrm{GHz}$ to $3.75 \mathrm{GHz}$ has obtained in triple stage topology. The gain of the triple stage impedance matching topology obtained as $-18 \mathrm{~dB},-33 \mathrm{~dB},-98 \mathrm{~dB}$, $-57 \mathrm{~dB}$ and $-31 \mathrm{~dB}$. The $\mathrm{Q}$ factor value for single, double and triple stage network has 2.048, 1.131 and 0.855 respectively. From the above $\mathrm{Q}$ factor value the band width is higher when the Q value is lower.

\section{References}

1. Ahmed E.Mahdi, Ayman G. Sobih and Magdy A. El-Kafafi, "Design and Implementation of $10 \mathrm{~W}$, Highly Linear, Wideband and Efficient Power Amplifier Using harmonic Termination", IEEE middle East Conference on Antenna and Propagation, 2016.

2. Yang Qian-kun, Liu Yuan-an, Yu Cui-ping, Li Shu- lan and $\mathrm{Li}$ Jiu-chao, "Design and implementation of a high-efficiency concurrent dual-band power amplifier", The Journal of China Universities of Posts and Telecommunications, 21, 6, 94-99, 2014.

3. Veeraiyah Thangasamy, Vinesh Thiruchelvam, Shaiful Jahari Hashim and Noor Ain Kamsani, "RF CMOS switch design methodologies for multiband transceiver applications", Pertanika Journals of Science \& Technology, 25, 2017.
4. Ji-Kang Nai, Yuan-Hung Hsiao, Yun-Shan Wang, Yu-Hsuan Lin and Huei Wang, "A 2.8-6 GHz High-Efficiency CMOS Power Amplifier with High-order Harmonic Matching Network", IEEE MTT-S International Microwave Symposium (IMS), 4, 2016.

5. Jhen-Ji Wanga, Duan-Yu Chena, San-Fu Wangb and Rong-Shan Wei, "A multi-band low noise amplifier with wide-band interference rejection improvement", International Journal of Electronics and Communications (AEÜ), 70, (320-325), 2016.

6. Xiangning Fan, Zhou Yu, Jiakai Lu and Zaijun Hua, "Design of a 0.7 1.5 GHz Wideband Power Amplifier in $0.18-\mu \mathrm{m}$ CMOS Process", MTT-S International Microwave Workshop Series on Advanced Materials and Processes for RF and THz Applications (IMWS-AMP), 978, 2015.

7. Jorge Julián Moreno and Edison Ferney Angarita, "A Wideband Output Matching Network for Doherty Power Amplifier Applications", 46th European Microwave Conference, 851-853, 2016.

8. Yu Qijin, Yu Cuiping, Tang Bihua and Liu Yuan'an, “ Design of High-Efficiency broadband power amplifier using low-pass bias network", The Journal of China Universities of Posts and Telecommunications, 23, 1, 91-96, 2016.

9. Jing Xia, Mengsu Yang and Anding Zhu, "Improved Doherty Amplifier Design with Minimum Phase Delay in Output Matching Network for Wideband Application", IEEE microwave and wireless components letters, Volume 26, 11, 915-917, 2016.

10. Pengliang Guo, Wa Kong, Jing Xia and Lixia Yang, "Wideband Doherty Power Amplifier Using Suitable Peaking Output Matching Network", Asia-Pacific Microwave conference, Volume 3, 2015 